\title{
The Determination of Barriers to Internal Rotation by Means of Electron Diffraction. Ferrocene and Ruthenocene
}

\author{
A. HAALA N D and J. E. NILSSON \\ Department of Chemistry, University of Oslo, Blindern, Oslo 3, Norway
}

\begin{abstract}
The electron scattering pattern from a molecule undergoing internal rotation is a function of an angular probability function $W(\varphi)$ defined in such a way that the probability of the angle of rotation being between $\varphi$ and $\varphi+\mathrm{d} \varphi$ is equal to $W(\varphi) \mathrm{d} \varphi$.

It is shown that if the restricting barrier is of the shape $V(\varphi)=\left(V_{0} / 2\right)(1-\cos \sigma \varphi)$, for low and intermediate values of $V_{0} / k T$ the angular probability function is given by the classical expression $W(\varphi)=N \exp (V(\varphi) / k T)$.

It is assumed that the angular probability function can be found from classical statistics for all shapes of the restricting barrier, a program for least-squares refinement of the barrier to internal rotation on electron diffraction data has been written, and the rotational barriers in ferrocene and ruthenocene have been refined.

The equilibrium conformation of the free ferrocene molecule is prismatic, the barrier to internal rotation is found to be $0.9 \pm 0.3$ kcal mole ${ }^{-1}$. The magnitude obtained for the barrier is fairly insensitive to the shape assumed for $V(\varphi)$. For ruthenocene the experimental data were not good enough to permit a determination of $V_{0}$, but the best agreement was obtained for a prismatic model.
\end{abstract}

$\mathrm{T}^{\mathrm{h}}$ The synthesis of a previously unknown compound of composition $\mathrm{FeC}_{10} \mathrm{H}_{10}$ was reported nearly simultaneously by two groups of workers in $1951.1,2$ Within months the correct - and entirely novel - molecular structure had been proposed, again independently and simultaneously by two groups, Fischer and Phab $^{3}$ in Germany, and Wilkinson, Rosenblum, Whiting and Woodward ${ }^{4}$ in the U.S.

The molecule consists of two regular $\mathrm{C}_{5} \mathrm{H}_{5}$ rings that lie one on top of the other, with the fivefold symmetry axes coinciding. The rings thus form a cage around the metal atom (Fig. 1). Depending on the relative orientation of the rings they form a pentagonal prism (A) or antiprism (B).

It is clear that whether the equilibrium conformation is prismatic or antiprismatic, the barrier to internal rotation must be quite low since all attempts to isolate rotational isomers of disubstituted compounds have been unsuccessful. 

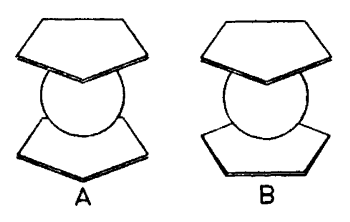

Fig. 1. Molecular models of ferrocene and ruthenocene; $A$, prismatic, and $B$, antiprismatic.
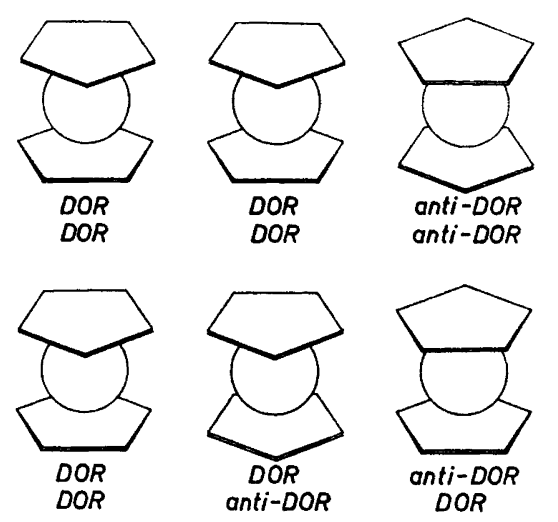

Fig. 2. Two mixtures of ferrocene molecules in different conformations, both compatible with the available data on ferrocene crys. tals at room temperature.

$\mathrm{Fe}\left(\mathrm{C}_{5} \mathrm{H}_{5}\right)_{2}$ crystallizes into spacegroup $P 2_{1} / a$ with two molecules per unit cell. ${ }^{3}$ Hence, if disorder could be disregarded, it would follow that each molecule has a center of symmetry and that - in the crystal at least - the molecule is antiprismatic.

The correctness of the "sandwich" model (Fig. 1) was proven by a complete $\mathrm{X}$-ray diffraction investigation by Dunitz, Orgel, and Rich. ${ }^{5}$ Electron density maps showed clear maxima at positions expected for carbon atoms in an antiprismatic molecule. It was noted, however, that difference synthesis gave peaks of high electron density in the middle of the $\mathrm{C}-\mathrm{C}$ bonds and strongly negative troughs at carbon atom positions.

Neutron diffraction measurements have later shown that ferrocene crystals in fact are highly disordered. ${ }^{6}$ As many as one third of the cyclopentadienyl rings appear to be rotated $36^{\circ}$ away from the position indicated by the maxima in the electron density maps (referred to as the DOR orientation in Fig. 2) into what might be called an anti-DOR orientation.

Once it is realized that the crystals are disordered, no conclusions regarding molecular symmetry can be drawn from the space group symmetry: The results of the X-ray and neutron diffraction experiments are compatible with a crystal consisting of two thirds anti-prismatic molecules with both rings in the DOR-orientation and one third antiprismatic molecules with both rings in the anti-DOR-orientation (Fig. 2A). But they are equally compatible with a crystal consisting of one third antiprismatic molecules and two thirds prismatic molecules (Fig. 2B).

Ferrocene has a $\lambda$ point transition at $163.9^{\circ} \mathrm{K} .^{7}$ The accompanying entropy change is $\Delta S=R \ln 1.89 \mathrm{cal} \mathrm{deg}^{-1} \mathrm{~mole}^{-1}$ and the enthalpy change $\Delta H=204$ cal mole $\mathrm{e}^{-1}$. It is probable that the transition is associated with introduction of disorder into the crystal. The structure of the - presumably ordered crystal below the transition point remains unknown as crystals are shattered when cooled below the transition point.? 


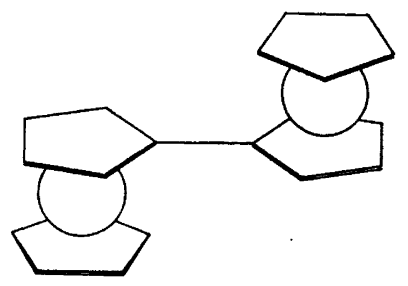

A

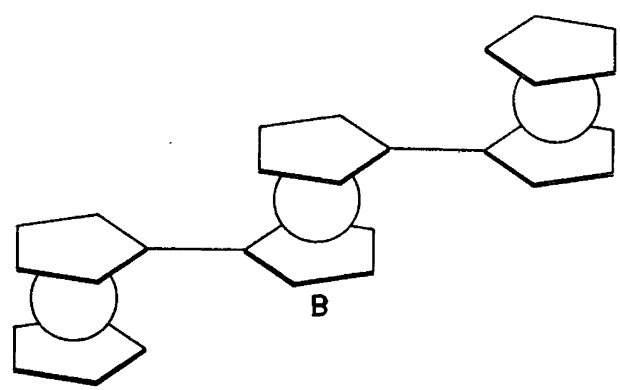

Fig. 3. Molecular conformations of diferrocyl (A) and terferrocyl (B) in the crystal.

No indications of disorder are found in X-ray studies of diferrocyl ${ }^{\mathbf{8 , 9}}$ and terferrocyl. ${ }^{10}$ In diferrocyl the two ferrocyl units are neither prismatic nor antiprismatic (Fig. 3A). The dihedral angle which would be $0^{\circ}$ in a prismatic and $36^{\circ}$ in an antiprismatic molecule, is $17^{\circ} .8,9$ In terferrocyl (Fig. 3B) the terminal ferrocyl-units are prismatic, while the central unit is antiprismatic. It is hard to escape the conclusion that the conformation of these two compounds - and accordingly the conformation of ferrocene too - in the crystal is determined by intermolecular as much as by intramolecular forces.

The barrier to internal rotation of the cyclopentadienyl rings in ferrocene crystals below the transition point has been measured by NMR techniques.11 The value found was $V=1.8 \pm 0.2 \mathrm{kcal} \mathrm{mole} \mathrm{m}^{-1}$. Again we may assume this energy to be the sum of inter- and intra-molecular terms.

A complete $X$-ray diffraction analysis of the ferrocene-analogue $\mathrm{Ru}\left(\mathrm{C}_{5} \mathrm{H}_{5}\right)_{2}$ or ruthenocene has been carried out by Hardgrove and Templeton. ${ }^{12}$ The spacegroup is Pnma, and there are four molecules per unit cell. Each molecule has a symmetry plane through the metal atom. Three dimensional leastsquares refinement gave a prismatic structure with the symmetry plane perpendicular to the ligand rings. No indication of disorder was found. The barrier to internal rotation of the ligand rings in the crystal below $250^{\circ} \mathrm{K}$ is $2.3 \pm 0.2 \mathrm{kcal} \mathrm{mole}^{-1} .11$

The infrared and Raman spectra of ferrocene and ruthenocene have been investigated by Lippincott and Nelson, ${ }^{13}$ the Raman spectrum of ferrocene by Stammreich. ${ }^{14}$ There is still no agreement on the assignment of all lines. It should be realized, however, that the point groups $D_{5 h}$ (prismatic model) and $D_{5 d}$ (antiprismatic) predict the same number and same kind of lines in the infrared and Raman spectra. ${ }^{15}$ Hence it has not been possible to distinguish the two models by spectroscopic means. The frequency of the normal mode associated with libration of the rings would give a clue to the magnitude of the barrier. But since the mode is both infrared and Raman inactive it has not yet been observed.

Ferrocene has been the subject of three (published) electron diffraction studies. Seibold and Sutton ${ }^{16}$ measured the scattering pattern from the gas at $400^{\circ} \mathrm{C}$ and found it to be consistent with a model with zero rotational barrier. A more recent investigation of gas at an unspecified temperature by a group

Acta Chem. Scand. 22 (1968) No. 8 
of Russian workers yielded the same conclusion. ${ }^{17}$ Bohn and Haaland, ${ }^{18}$ however, found that the scattering pattern from the gas at $140^{\circ} \mathrm{C}$ was consistent only with a prismatic molecular structure. They estimated the barrier to internal rotation in the free molecule to be $V_{0}=1.1 \mathrm{kcal} \mathrm{mole}^{-1}$.

\section{EXPERIMENTAL AND CALCULATION PROCEDURE}

The electron scattering pattern from gaseous ferrocene and ruthenocene were recorded on the Oslo electron diffraction units. ${ }^{19,20}$ The nozzle temperature for both compounds was $140^{\circ} \pm 10^{\circ} \mathrm{C}$. With ferrocene, exposures were made with nozzle to photographic plate distances of about $15 \mathrm{~cm}, 48 \mathrm{~cm}$, $*$ and 130 $\mathrm{cm}$. With ruthenocene, exposures were made with the distances $19 \mathrm{~cm}, 48 \mathrm{~cm}$, and $130 \mathrm{~cm}$. For each distance four plates were photometered. Optical densities were read off at $\Delta s=0.25 \AA^{-1}$ or $\Delta s=0.125 \AA^{-1}$ intervals and processed in the usual way. ${ }^{20}(s=4 \pi \sin (\vartheta / 2) / \lambda$, when $\vartheta$ is the diffraction angle and $\lambda$ the electron wavelength).

A curve drawn through the experimental modified molecular intensity points of ferrocene is shown in Fig. 4A. The data extend from $s=0.50 \AA^{-1}$ to $53.75 \AA^{-1}$. Below $s=7 \AA^{-1}$ the point density is 8 points per $\AA^{-1}$, above $s=7 \AA^{-1}$ the density is 4 points per $\AA^{-1}$.

A curve drawn through the experimental modified molecular intensity points of ruthenocene is shown in Fig. 5A. The data extend from $s=0.50 \AA^{-1}$ to $46.00 \AA^{-1}$. Below $s=15 \AA^{-1}$ the point density is 8 points per $\AA^{-1}$ above $s=15 \AA^{-1}$ it is 4 points per $\AA^{-1}$.

Complex atomic scattering factors, $f(s)=|f(s)| \exp (i \eta(s))$, were computed by the partial wave expansion method with a program written by Peacher. ${ }^{21}$

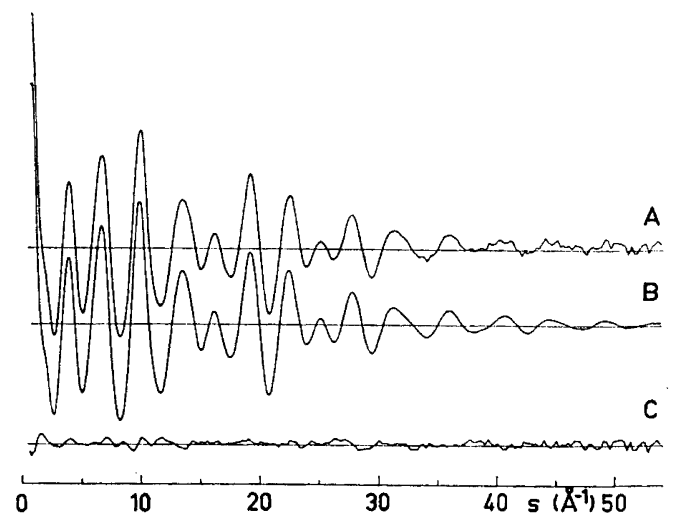

Fig. 4. Modified molecular intensity curves of ferrocene. A, experimental, B, theoretical, calculated from the parameter values of Table 1 , and C, difference curve.

\footnotetext{
* This set of plates was also used by Bohn and Haaland. ${ }^{18}$ The $19 \mathrm{~cm}$ plates used by these authors, however, were found to be flawed by a slight error in the electron wavelength and discarded. The bond distances in ferrocene obtained by us (Table 1) are therefore some $0.7 \%$ greater than those reported by Bohn and Haaland.
} 


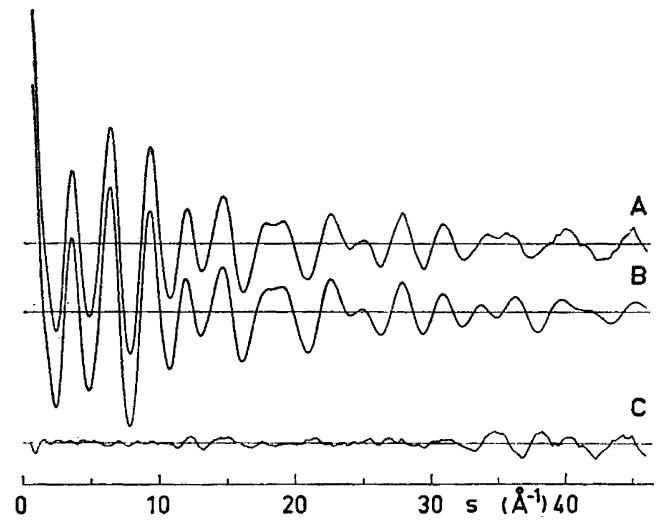

Fig. 5. Modified molecular intensity curves of ruthenocene. A, experimental, B, theoretical, calculated from the parameter values of Table 1, and C, difference curve.

The scattering potentials of carbon ${ }^{22,24}$ and iron ${ }^{23,24}$ have been found by non-relativistic Hartree-Fock, the scattering potential of ruthenium ${ }^{25,26}$ by relativistic Hartree-Fock-Slater computations.

Theoretical intensity curves were calculated from

$$
\begin{gathered}
I^{\mathrm{cc}}(s)=\operatorname{const} \sum_{i \neq j} \frac{\left|f_{i}(s)\right|\left|f_{j}(s)\right|}{\left|f_{\mathrm{C}}(s)\right|\left|f_{\mathrm{C}}(s)\right|} \cos \left(\eta_{i}(s)-\eta_{j}(s)\right) \frac{\sin \left(R_{i j} s\right)}{R_{i j}} \exp \left(-\frac{1}{2} u_{i j}{ }^{2} s^{2}\right) \\
=\operatorname{const} \sum_{i \neq j} g_{i j / \mathrm{CC}}(s) \frac{\sin \left(R_{i j} s\right)}{R_{i j}} \exp \left(-\frac{1}{2} u_{i j}{ }^{2} s^{2}\right)
\end{gathered}
$$

The sum extends over all atom pairs $i, j$ in the molecule. $R_{i j}$ is the internuclear distance, $u_{i j}$ the root mean square amplitude of vibration.

Radial distribution (RD) curves were obtained by Fourier inversion of the experimental and theoretical intensity curves after multiplication with

Fig. 6. Radial distribution curves of ferrocene. A, experimental, $B$, theoretical, calculated from the parameter values obtained by refinement of a prismatic model (Table 1), and $\mathrm{C}$, theoretical calculated from the parameter values obtained by refinement of an antiprismatic model. The experimental curve is stippled in in B and C. Artificial damping constant $k=0.001 \AA^{2}$.

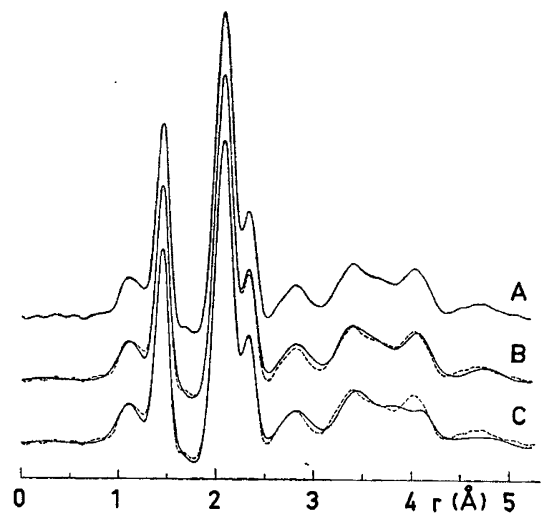




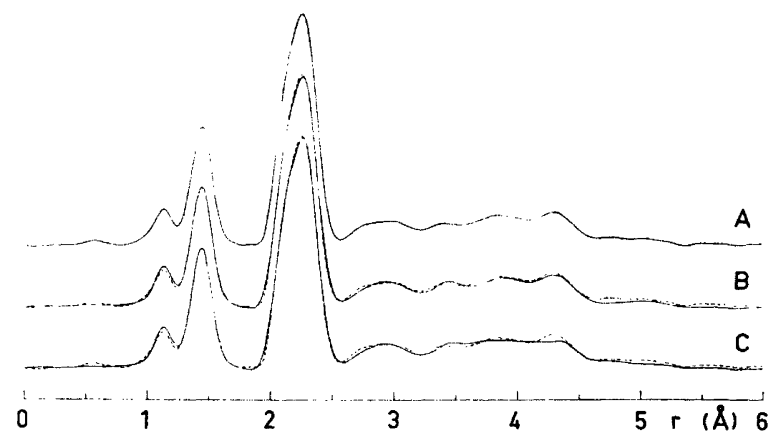

Fig. \%. Radial distribution curves of ruthenocene. A, experimental, B, theoretical, calculated from the parameter values obtained by refinement of a prismatic model (Table 1), and C, theoretical, calculated from the parameter values obtained by refinement of an antiprismatic model. The experimental curve is stippled in in B and C. Artificial damping constant $k=0.002 \AA^{2}$.

the artificial damping function $\exp \left(-k s^{2}\right) \cdot{ }^{20}$ An experimental RD curve of ferrocene is shown in Fig. 6A, an experimental RD curve of ruthenocene in Fig. 7A.

The molecular structures were refined by least-squares calculations on the intensity data. ${ }^{27,28}$

\section{DETERMINATION OF BOND DISTANCES}

The molecular structure of ferrocene was first refined by least-squares calculations on the intensity data under the assumption that the molecule is prismatic. The resulting square-error sum was 1.77. The structure was then refined under the assumption that the molecule is antiprismatic. The resulting square-error sum was 2.60 . (The absolute magnitude of these numbers is arbitrary, only their relative magnitude has any significance). For ruthenocene the square-error sum obtained was 1.65 for the prismatic and 1.86 for the antiprismatic model. Hence Hamilton's test ${ }^{29}$ indicates that the antiprismatic model can be discarded at the 0.005 significance level for both molecules.* We should, however, like to strike a note of caution at this point; Hamilton's test presupposes that the proper weighting-scheme has been used and that the observational equations are linear. These conditions may not be entirely satisfied.

The values obtained for interatomic distances and vibrational amplitudes for the prismatic models are listed in Table 1. The values obtained for the interatomic distances and vibrational amplitudes unaffected by the relative motion of the rings are nearly identical to those obtained by refinement on an antiprismatic model. They are therefore correct regardless of the symmetry of the equilibrium conformation.

* The same applies to dibenzene chromium. Least-squares refinement on the data recorded by Haaland ${ }^{30 a}$ gives a square-error sum of 0.94 for the prismatic and 1.09 for the antiprismatic model. ${ }^{\mathrm{sob}}$ 
Table 1. Bond distances and root mean square vibrational amplitudes of ferrocene and ruthenocene obtained by refinement on the prismatic models. The standard deviations include the effect of errors in the electron wavelength. Distances are given as $r_{g}(1) .^{33}$

\begin{tabular}{lll} 
& $\mathrm{Fe}\left(\mathrm{C}_{5} \mathrm{H}_{5}\right)_{2}$ & \multicolumn{1}{c}{$\mathrm{Ru}\left(\mathrm{C}_{5} \mathrm{H}_{5}\right)_{2}$} \\
$R(\mathrm{C}-\mathrm{C})$ & $1.440 \pm 0.002$ & $1.439 \pm 0.002$ \\
$R(\mathrm{C}-\mathrm{H})$ & $1.104 \pm 0.006$ & $1.130 \pm 0.006$ \\
$R(\mathrm{M}-\mathrm{C})$ & $2.064 \pm 0.003$ & $2.196 \pm 0.003$ \\
& & \\
$u(\mathrm{C}-\mathrm{C})$ & $0.046 \pm 0.001$ & $0.051 \pm 0.001$ \\
$u(\mathrm{C}-\mathrm{H})$ & $0.092 \pm 0.006$ & $0.012 \pm 0.038$ \\
$u(\mathrm{M}-\mathrm{C})$ & $0.062 \pm 0.001$ & $0.060 \pm 0.001$ \\
$u\left(\mathrm{M} \cdots \mathrm{H}^{\prime}\right)$ & $0.124 \pm 0.009$ & $0.131 \pm 0.013$ \\
$u\left(\mathrm{C}_{1} \cdots \mathrm{C}_{3}\right)$ & $0.054 \pm 0.002$ & 0.09 (ass) \\
$u\left(\mathrm{C}_{1} \cdots \mathrm{H}_{2}\right)$ & $0.145 \pm 0.015$ & 0.09 (ass) \\
$u\left(\mathrm{C}_{1} \cdots \mathrm{H}_{3}\right)$ & $0.158 \pm 0.017$ & $0.244 \pm 0.033$ \\
$u\left(\mathrm{C}_{1} \cdots \mathrm{C}_{1}{ }^{\prime}\right)$ & $0.101 \pm 0.008$ & $0.177 \pm 0.036$ \\
$u\left(\mathrm{C}_{1} \cdots \mathrm{C}_{2}{ }^{\prime}\right)$ & $0.193 \pm 0.019$ & $0.123 \pm 0.036$ \\
$u\left(\mathrm{C}_{1} \cdots \mathrm{C}_{3}{ }^{\prime}\right)$ & $0.117 \pm 0.020$ & 0.19 (ass) \\
$u\left(\mathrm{C}_{1} \cdots \mathrm{H}_{1}\right)$ & 0.15 (ass) & 0.21 (ass) \\
$u\left(\mathrm{C}_{1} \cdots \mathrm{H}_{2}\right)$ & 0.24 (ass) & 0.16 (ass) \\
$u\left(\mathrm{C}_{1} \cdots \mathrm{H}_{3}\right)$ & 0.16 (ass) &
\end{tabular}

Theoretical modified molecular intensity curves calculated from the parameter values in Table 1 are shown in Figs. 4B (ferrocene) and 5B (ruthenocene).

Fig. 6B shows a theoretical RD curve of ferrocene calculated from the parameters obtained by refinement on a prismatic model (Table 1). An RD curve calculated from the parameter values obtained by refinement on an antiprismatic model is shown in Fig. 6C. It is seen that in the region of distances between atoms in different ligand rings $(3-5 \AA)$ a much better agreement has been obtained for the prismatic model.

The corresponding curves for ruthenocene are shown in Fig. 7B and C. The best agreement is still obtained for the prismatic model, but the difference is much smaller than in ferrocene.

The vibrational amplitude of the $\mathrm{M}-\mathrm{C}$ bond in ferrocene and ruthenocene is less than half the $\mathrm{M}-\mathrm{C}$ amplitude in the more unstable compounds manganese dicyclopentadienide ${ }^{31}(0.135 \pm 0.002 \AA)$, dicyclopentadienyllead ${ }^{32}$ $(0.142 \pm 0.004 \AA)$, and dicyclopentadienyltin ${ }^{32}(0.157 \pm 0.009 \AA)$. That the metal-to-ligand bonding is weak in these compounds is illustrated by the

Table 2. Carbon-carbon bond distances in biscyclopentadienylmetal complexes as determined by electron diffraction.

$\begin{array}{lll} & & R(\mathrm{C}-\mathrm{C})(\AA) \\ \mathrm{Fe}\left(\mathrm{C}_{5} \mathrm{H}_{5}\right)_{2} & \text { (this work) } & 1.440 \pm 0.002 \\ \mathrm{Ru}\left(\mathrm{C}_{5} \mathrm{H}_{5}\right)_{2} & \text { (this work) } & 1.439 \pm 0.002 \\ \mathrm{Mn}\left(\mathrm{C}_{5} \mathrm{H}_{5}\right)_{2} & \text { (Ref. 31) } & \\ \mathrm{Pb}\left(\mathrm{C}_{5} \mathrm{H}_{5}\right)_{2} & \text { (Ref. 32) } & 1.429 \pm 0.002 \\ \mathrm{Sn}\left(\mathrm{C}_{5} \mathrm{H}_{5}\right)_{2} & \text { (Ref. 32) } & 1.430 \pm 0.002 \\ \end{array}$

Acta Chem. Scand. 22 (1968) No. 8 
fact that the $\mathrm{M}-\mathrm{C}$ bond is more than $0.3 \AA$ longer in $\mathrm{Mn}\left(\mathrm{C}_{5} \mathrm{H}_{5}\right)_{2}$ than in $\mathrm{Fe}\left(\mathrm{C}_{5} \mathrm{H}_{5}\right)_{2}$.

The $\mathrm{C}-\mathrm{C}$ bond distances found in the five compounds are set out in Table 2. It would appear that the $\mathrm{C}-\mathrm{C}$ bond is about $0.01 \AA$ shorter in the compounds where the metal-to-ligand bonds are weak.

\section{MOLECULAR INTENSITY FOR A MOLECULE UNDERGOING INTERNAL ROTATION}

The modified molecular intensity for a nonvibrating molecule is given by

$$
I(s)=\sum_{i \neq j} g_{i j}(s) \sin \left(R_{i j}{ }^{0} s\right) / R_{i j}{ }^{0}
$$

$\boldsymbol{R}_{i j}{ }^{0}$ is the (equilibrium) distance between the nuclei of atoms $i$ and $j$, and the sum extends over all atom pairs in the molecule. (The function $g_{i j}$ is defined as in eqn. 1).

If the molecule vibrates, it is convenient to define a radial probability function, ${ }^{33} P_{i j}(R)$, such that the probability of the distance between nuclei $i$ and $j$ being between $R$ and $R+\mathrm{d} R$ (at any particular instant) is $P_{i j}(R) \mathrm{d} R$. The modified molecular intensity from a large number of molecules is then given by

$$
I(s)=\sum_{i \neq j} g_{i j}(s) \int_{0}^{\infty} P_{i j}(R) \sin (R s) / R \mathrm{~d} R
$$

If $P_{i j}(R)$ is Gaussian,

$$
P_{i j}(R)=\left(2 \pi u_{i j}{ }^{2}\right)^{-\frac{1}{2}} \exp \left[-\left(R-R_{i j}{ }^{0}\right)^{2} / 2 u_{i j}{ }^{2}\right]
$$

where $u_{i j}$ is the root-mean-square variation (amplitude of vibration) of $R_{i j}$,

$$
I(s)=\sum_{i \neq j} g_{i j}(s) \sin \left(R_{i j} s\right) / R_{i j} \exp \left(-\frac{1}{2} u_{i j}{ }^{2} s^{2}\right)
$$

where $R_{i j}$ to a very high degree of precision is given by ${ }^{33}$

$$
R_{i j}=R_{i j}{ }^{0}-u_{i j}{ }^{2} / R_{i j}{ }^{0}
$$

The distance between the nuclei of atoms $i$ and $j$ is determined by the equilibrium distance $\left(R_{i j}{ }^{0}\right)$ and by the magnitude of the normal (displacement) coordinates $(\bar{Q})$ :

$$
R_{i j}=F_{i j}\left(R_{i j}^{0}, \bar{Q}\right)
$$

For sufficiently small displacements, higher powers in the normal coordinates may be neglected and

$$
R_{i j}=R_{i j}{ }^{0}+\sum_{k=1}^{K} c_{i j k} Q_{k}
$$

The sum extends over all $K$ normal coordinates.

If $\left\{\psi_{k, i}\right\}$ are the wavefunctions of the stationary states of the $k$ 'th normal Acta Chem. Scand. 22 (1968) No. 8 
mode, the probability of the $k^{\prime}$ th normal coordinate being between $Q$ and $Q+\mathrm{d} Q$ is

$$
W_{k}(Q) \mathrm{d} Q=\sum \alpha_{k, i}\left|\psi_{k, i}(Q)\right|^{2} \mathrm{~d} Q
$$

where $\left\{\alpha_{k}, i\right\}$ are the normalized Boltzmann coefficients.

If the normal mode is harmonic it follows from (9) that ${ }^{34}$

$$
W_{k}(Q) \mathrm{d} Q=\left(2 \pi u_{k}^{2}\right)^{-\frac{1}{2}} \exp \left(-Q^{2} / 2 u_{k}^{2}\right) \mathrm{d} Q
$$

$u_{k}$ is the root-mean-square variation of $Q_{k}$. It is determined by the force constant $\lambda_{k}{ }^{\prime}$ and the energy associated with the mode:

$$
\lambda_{k}{ }^{\prime} u_{k}{ }^{2}=\sum_{i} \alpha_{k, i} \varepsilon_{k, i}
$$

It is now possible to determine the radial probability functions:

$$
P_{i j}\left(R_{i j}\right) \mathrm{d} R_{i j}=\int \prod_{k=1}^{K} W_{k}\left(Q_{k}\right) \mathrm{d} Q_{k}
$$

or if all displacements are sufficiently small

$$
\begin{aligned}
& P_{i j}\left(R_{i j}\right) \mathrm{d} R_{i j}=\int_{Q_{2}} \ldots \int_{Q_{k}} W_{1}\left(\left(R_{i j}-R_{i j}{ }^{0}-\sum_{k=2}^{K} c_{i j k} Q_{k}\right) / c_{i j 1}\right) \\
& \mathrm{d} Q_{1} \cdot \underset{k=2}{K} W_{k}\left(Q_{k}\right) \mathrm{d} Q_{k}
\end{aligned}
$$

If all modes are harmonic $W_{k}$ is given by eqn. (10) and the integration is easily carried out. Keeping in mind that $\mathrm{d} Q_{1}=\mathrm{d} R_{i j} / c_{i j 1}$ one obtains:

where

$$
P_{i j}\left(R_{i j}\right) \mathrm{d} R_{i j}=\left(2 \pi u_{i j}{ }^{2}\right)^{-\frac{1}{2}} \exp \left[-\left(R_{i j}-R_{i j}{ }^{0}\right)^{2} / 2 u_{i j}{ }^{2}\right] \mathrm{d} R_{i j}
$$

$$
u_{i j}^{2}=\sum_{k=1}^{K} c_{i j k}^{2} u_{k}^{2}
$$

If the molecule undergoes internal rotation against a small restricting barrier, the method just outlined must be somewhat modified. We shall assume that the libration is a normal mode. This will for instance be the case in ethane-like molecules where internal rotation is in a symmetry class by itself. In ferrocene internal rotation is in the same class as an in-plane in-phase deformation of all $\mathrm{C}-\mathrm{H}$ bonds. In this case, however, one may expect that the separation works on physical grounds; the $\mathrm{C}-\mathrm{H}$ deformation frequency is found at about $1200 \mathrm{~cm}^{-115}$ the frequency of libration is expected below $100 \mathrm{~cm}^{-1}$.

The probability of the angle of internal rotation being between $\varphi$ and $\varphi+d \varphi$ is then given by an angular probability function $W_{K}(\varphi)$ that in the general case will not be Gaussian. Secondly, for distances that vary greatly with the angle of internal rotation (such as the distances between carbon atoms in different ligand rings in ferrocene) eqn. (8) is no longer sufficiently accurate. 
Rather

$$
R_{i j}=R_{i j}(\varphi)+\sum_{k=1}^{K-1} c_{i j k}(\varphi) Q_{k}
$$

where $R_{i j}(\varphi)$ is the internuclear distance found in a molecule with dihedral angle $\varphi$ and all other displacement coordinates equal to zero.

The radial probability function for such a distance is

$$
\begin{gathered}
P_{i j}\left(R_{i j}\right) \mathrm{d} R_{i j}= \\
\int_{Q_{2}} \cdots \int_{Q_{K-1}} \int_{\varphi} W_{1}\left(\left(R_{i j}-R_{i j}(\varphi)-\sum_{k=2}^{K-1} c_{i j k}(\varphi) Q_{k}\right) / c_{i j 1}(\varphi)\right) \mathrm{d} Q_{1} \\
\prod_{k=2}^{K-1}\left(W_{k}\left(Q_{k}\right) \mathrm{d} Q_{k}\right) W_{K}(\varphi) \mathrm{d} \varphi
\end{gathered}
$$

If it is assumed that all modes except libration are harmonic, introduction of eqn. 10 and integration over $Q_{2} \ldots Q_{K-1}$ gives:

$$
\begin{gathered}
P_{i j}\left(R_{i j}\right) \mathrm{d} R_{i j}= \\
\int_{\varphi=0}^{2 \pi}\left(2 \pi u_{\mathrm{f}}^{2}\right)^{-\frac{1}{2}} \exp \left[-\left(R_{i j}-R_{i j}(\varphi)\right)^{2} / 2 u_{\mathrm{f}}^{2}(\varphi)\right] W_{K}(\varphi) \mathrm{d} \varphi \mathrm{d} R_{i j} \\
u_{\mathrm{f}}^{2}(\varphi)=\sum_{k=1}^{K-1} c_{i j k}^{2}(\varphi) u_{k}^{2}
\end{gathered}
$$

where

$u_{\mathrm{f}}$ is the root-mean-square amplitude of vibration resulting from all modes of vibration except libration. It is often referred to as "the framework amplitude". In an excellent paper in 1958, Morino and Hirota ${ }^{35}$ showed how it could be calculated for $\mathrm{Si}_{2} \mathrm{Cl}_{6}$.

The contribution to the molecular intensity for a large number of molecules from the distance $R_{i j}$ is

$$
\begin{gathered}
I_{i j}(s)=g_{i j}(s) \int_{\varphi} \int_{R_{i j}}\left(2 \pi u_{\mathrm{f}}^{2}(\varphi)\right)^{-\frac{1}{2}} \exp \left[-\left(R_{i j}-R_{i j}(\varphi)\right)^{2} / 2 u_{\mathrm{f}}^{2}(\varphi)\right] \\
W_{K}(\varphi) \sin \left(R_{i j} s\right) / R_{i j} \mathrm{~d} R_{i j} \mathrm{~d} \varphi
\end{gathered}
$$

and integration over $R_{i j}$ gives

$$
I_{i j}(s)=g_{i j}(s) \int_{\varphi} W_{K}(\varphi) \sin \left(R_{i j}(\varphi) s\right) / R_{i j}(\varphi) \exp \left(-\frac{1}{2} u_{\mathrm{f}}^{2}(\varphi) s^{2}\right) \mathrm{d} \varphi
$$

It is seen that the scattering pattern is that expected from a mixture of hypothetical molecules with different equilibrium dihedral angles, each undergoing small amplitude vibrations $\left(u_{\mathrm{f}}\right)$ about the equilibrium conformation. 


\section{THE ANGULAR PROBABILITY FUNCTION CALCULATED FROM QUANTUM} MECHANICS AND FROM CLASSICAL STATISTICAL MECHANICS

If the molecule consists of two symmetrical tops which are joined in such a way that their unique rotational axes coincide, internal rotation can be separated from the overall rotation of the molecule. The Schrödinger equation for the mode of internal rotation is then ${ }^{36}$

$$
\left(\hbar^{2} / 2 I\right) \frac{\mathrm{d}^{2} \psi(\varphi)}{\mathrm{d} \varphi^{2}}+(E-V(\varphi)) \psi(\varphi)=0
$$

$V(\varphi)$ is the restricting potential, and $I$ is the reduced moment of inertia of the system $\left(I=I_{1} I_{2} /\left(I_{1}+I_{2}\right)\right)$.

We shall assume that the potential is of the form

$$
V(\varphi)=\left(V_{0} / 2\right)(1-\cos \sigma \varphi)
$$

where $\sigma$ is the symmetry number of the two tops. If the following definitions are made

eqn. (23) becomes

$$
\begin{gathered}
x=\frac{1}{2} \sigma \varphi \\
\mathrm{M}(x)=\psi(\varphi) \\
a=32 \\
\pi^{2} I\left(E-V_{0} / 2\right) /\left(\begin{array}{ll}
\sigma^{2} & \hbar^{2}
\end{array}\right) \\
q=\pi^{2} I V_{0} /\left(\sigma^{2} \hbar^{2}\right)
\end{gathered}
$$

$$
\frac{\mathrm{d}^{2} \mathrm{M}(x)}{\mathrm{d} x^{2}}+(a+16 q \cos 2 x) \mathrm{M}(x)=0
$$

The solutions to this equation are the Mathieu functions.

The equation can be easily solved in two cases:

a) $16 q \ll a$;

$$
\begin{gathered}
\frac{\mathrm{d}^{2} \mathrm{M}(x)}{\mathrm{d} x^{2}}+a \mathrm{M}(x)=0 \\
\mathrm{M}(x)=\left\{\begin{array}{l}
\sin \sqrt{a x} \\
\cos \sqrt{a x}
\end{array}\right.
\end{gathered}
$$

and because of the periodicity of $\mathrm{M}(x)$

$$
a=4 m^{2}, m=0,1,2, \cdots
$$

b) $q$ very large

$$
\frac{\mathrm{d}^{2} \mathrm{M}(x)}{\mathrm{d} x^{2}}+\left(a+16 q-32 q x^{2}\right) \mathrm{M}(x)=0
$$

This is the Schrödinger equation for the harmonic oscillator and

$$
a=32 q(2 m+1)-16 q, m=0,1,2, \cdots
$$

Goldstein ${ }^{37}$ has shown how the approximate eigenvalues obtained from the limiting forms of the differential equation can be successively refined, and Acta Chem. Scand. 22 (1968) No. 8 
how the wavefunctions can be obtained from the refined eigenvalues. Using the method outlined by him we have solved eqn. (25) for values of $q$ ranging from 5 to 45 in steps of 5 . For each value of $q\left(V_{0}\right)$ the fifty lowest eigenvalues and the corresponding wavefunctions were determined, and an angular probability function computed according to

$$
W(\varphi)=N \sum_{i=1}^{50} \exp \left(-E_{i} / k T\right) \psi_{i}{ }^{2}(\varphi)
$$

$N$ is a normalizing constant such that

$$
\int_{0}^{2 \pi} W(\varphi) d \varphi=1
$$

$T=413^{\circ} \mathrm{K}$, the temperature of our experiment. For $q=5,\left(E_{50}-E_{1} / k T=16\right.$, for $q=45,\left(E_{50}-E_{1}\right) / k T=20$. Moreover it is seen from eqn. (26) that the distance between energy levels increases with increasing energy. The series termination error should therefore be negligible. The angular probability functions of ferrocene obtained for $q=5\left(V_{0}=0.809 \mathrm{kcal} \mathrm{mole}^{-1}\right)$ and $q=45$ $\left(V_{0}=7.379 \mathrm{kcal}^{\mathrm{mole}}{ }^{-1}\right)$ are plotted in Fig. 8 .

According to classical statistical mechanics the angular probability function should be given by

$$
W_{\mathrm{ci}}(\varphi)=N^{\prime} \exp (-V(\varphi) / k T)
$$

We have plotted the probability function obtained in this manner along with the one obtained from quantum mechanics for ferrocene with barriers varying from 0.8 to $7.4 \mathrm{kcal} \mathrm{mole}^{-1}$. When drawn on the scale of Fig. 8 they cannot be distinguished.

Furthermore, starting with the quantum mechanical probability function we have sought the classical function

$$
W_{\mathrm{cl}}{ }^{\prime}(\varphi)=N^{\prime} \exp \left(-V^{\prime}(1-\cos 5 \varphi) / 2 k T\right)
$$

that gave the best least-squares fit. It was found that in all cases $V_{0}$ and $V^{\prime}$ differed by less than $2 \%$. We have not sought to investigate whether this difference is real or whether it could be removed by increasing the accuracy of the computer computations. Since the barrier height for ferrocene found below have standard deviations amounting to about $30 \%$ of the total value, the following conclusion may be drawn: With the present accuracy of electron diffraction determination of barrier heights the angular probability function corresponding to a restricting potential of the form

$$
V=\left(V_{0} / 2\right)(1-\cos (\sigma \varphi))
$$

will for low and intermediate values of $V_{0} / k T$ be given with sufficient accuracy by the classical expression

$$
W(\varphi)=N \exp (-V(\varphi) / k T)
$$




\section{LEAST-SQUARES REFINEMENT OF THE BARRIER TO INTERNAL ROTA- TION}

A programme for least-squares refinement of the barrier to internal rotation in ferrocene-like molecules was written. The contribution to the theoretical intensity was calculated according to eqn. (1) for all atom pairs except C... $\mathrm{C}^{\prime}$ pairs with one carbon atom on each ligand ring. The interatomic distances and vibrational amplitudes of these pairs were given the values obtained by least-squares refinement on a prismatic model (Table 1) and not refined further.

The contribution to the theoretical intensity for each $\mathrm{C} \cdots \mathrm{C}^{\prime}$ pair was computed according to

$$
I_{\mathrm{CC}}(s)=\int_{\varphi=0}^{2 \pi} W(\varphi) \sin \left(R_{\mathrm{CC}}(\varphi) s\right) / R_{\mathrm{CC}^{\prime}}(\varphi) \exp \left(-\frac{1}{2} u_{\mathrm{f}}^{2}(\varphi) s^{2}\right) \mathrm{d} \varphi
$$

$\left(g_{\mathrm{cc} / \mathrm{cc}}(s)=1\right)$. The angle $\varphi$ was selected equal to zero when the two atoms are eclipsed.

The internuclear distance $R(\varphi)$ was calculated from the radius of the ligand rings, $r$, and the perpendicular distance between the rings, $H$;

$$
R_{\mathrm{CC}}{ }^{2}(\varphi)=H^{2}+2 r^{2}(1-\cos \varphi)
$$

$r$ and $H$ were first computed from the CC and MC bond distances, but in a final refinement $H$ was treated as an independent variable.

Morino and Hirota ${ }^{35}$ have calculated and plotted the framework vibrational amplitude, $u_{\mathrm{f}}(\varphi)$, for the $\mathrm{Cl} \cdots \mathrm{Cl}^{\prime}$ distance in $\mathrm{Si}_{2} \mathrm{Cl}_{6}$. The curve suggests a variation of the form

$$
u_{1}(\varphi)=C_{1}+D_{1} \cos \varphi
$$

The angular probability function was first assumed to have the form

$$
W_{1}(\varphi)=N \exp \left[A_{1}\left(\cos 5 \varphi+\beta_{1} \cos 10 \varphi\right)\right]
$$

If it is legitimate to use classical statistical mechanics (as we have shown it is if $\beta_{1}=0$ ), this probability function corresponds to a potential barrier of the form

with

$$
V(\varphi)=\left(V_{0} / 2\right)\left(1-\cos 5 \varphi-\beta_{1} \cos 10 \varphi\right)
$$

$$
V_{0} / k T=2 A_{1}
$$

Hence $A_{1}>0$ corresponds to a prismatic equilibrium conformation, and $A_{1}<0$ to an antiprismatic equilibrium conformation. The parameter $\beta_{1}$ determines the shape of the barrier: if $\beta_{1}<0$ the potential energy minimum near $\varphi=0^{\circ}$ becomes broader and the maximum at $\varphi=36^{\circ}$ more narrow, if $\beta_{1}>0$ the minimum becomes more narrow and the maximum broader than when $\beta_{1}=0$. See Fig. 9.

Unfortunately it proved impossible to refine this parameter. Instead $A_{1}, C_{1}$, and $D_{1}$ were refined simultaneously by least-squares for a set of fixed values of $\beta_{1}$. The resulting square-error sums and the values and standard deviations 
Table 3. Determination of the barrier to internal rotation in ferrocene. $W(\varphi)=N$ $\exp \left[A_{1}\left(\cos 5 \varphi+\beta_{1} \cos 10 \varphi\right)\right]$ and $u_{\mathrm{f}}(\varphi)=C_{1}+D_{1} \cos \varphi$. The results of simultaneous leastsquares refinement of $A_{1}, C_{1}$ and $D_{1}$ for a series of values of $\beta_{1}$.

\begin{tabular}{|c|c|c|c|c|c|c|}
\hline$\beta_{1}$ & $\begin{array}{l}\text { Square } \\
\text { error sum }\end{array}$ & $A_{1}$ & $C_{1}(\AA)^{a}$ & $D_{1}(\AA)^{a}$ & $V_{0} / k T$ & $V_{0}\left(\mathrm{kcal} \mathrm{mole}^{-1}\right)$ \\
\hline-0.25 & 2.08 & 0.61 & 0.110 & -0.004 & $1.21 \pm 0.48$ & $0.99 \pm 0.39$ \\
\hline-0.20 & 2.08 & 0.61 & 0.110 & -0.004 & $1.21 \pm 0.46$ & $0.99 \pm 0.38$ \\
\hline-0.15 & 2.09 & 0.59 & 0.110 & -0.004 & $1.17 \pm 0.44$ & $0.96 \pm 0.36$ \\
\hline-0.10 & 2.09 & 0.58 & 0.110 & -0.004 & $1.16 \pm 0.43$ & $0.95 \pm 0.35$ \\
\hline-0.05 & 2.09 & 0.57 & 0.110 & -0.004 & $1.13 \pm 0.41$ & $0.93 \pm 0.34$ \\
\hline 0.00 & 2.09 & 0.56 & 0.110 & -0.004 & $1.11 \pm 0.39$ & $0.91 \pm 0.32$ \\
\hline 0.05 & 2.09 & 0.55 & 0.110 & -0.004 & $1.07 \pm 0.38$ & $0.88 \pm 0.31$ \\
\hline 0.10 & 2.09 & 0.54 & 0.110 & -0.004 & $1.06 \pm 0.37$ & $0.87 \pm 0.30$ \\
\hline 0.15 & 2.09 & 0.53 & 0.110 & -0.004 & $1.05 \pm 0.35$ & $0.86 \pm 0.29$ \\
\hline 0.20 & 2.09 & 0.52 & 0.110 & -0.004 & $1.02 \pm 0.35$ & $0.84 \pm 0.29$ \\
\hline 0.25 & 2.09 & 0.50 & 0.110 & -0.004 & $0.99 \pm 0.34$ & $0.81 \pm 0.28$ \\
\hline
\end{tabular}

a Standard deviation $=0.007-0.008 \AA$.

of the three parameters are set out in Table 3. Three of the angular probability functions obtained are shown in Fig. 9.

In order to investigate how sensitive the results are to a qualitatively different kind of deviation from the simple $\left(\beta_{1}=0\right)$ cosine form, refinements were also carried out with the angular probability function

$$
\begin{cases}N\left[1+A_{2}\left(\frac{\pi-10 \varphi}{\pi}\right)^{1 / \beta_{2}}\right] ; & 0 \leq \varphi \leq \frac{\pi}{10} \\ N\left[1-A_{2}\left(\frac{10 \varphi-\pi}{\pi}\right)^{1 / \beta_{2}}\right] ; & \frac{\pi}{10}<\varphi \leq \frac{\pi}{5}\end{cases}
$$

From classical statistical mechanics it follows that

$$
V_{0} / k T=\ln \frac{1+A_{2}}{1-A_{2}}
$$

Again $A_{2}>0$ corresponds to a prismatic, and $A_{2}<0$ to an antiprismatic equilibrium conformation, while the shape of the barrier is determined by the parameter $\beta_{2}$. The result of least-squares refinements of $A_{2}, C_{1}$, and $D_{1}$ for a set of fixed values of $\beta_{2}$ are listed in Table 4. Three of the angular probability functions obtained are shown in Fig. 10.

It is seen from the tables that the square-error sum is very nearly the same for both sets of refinements and for all values of $\beta_{1}$ and $\beta_{2}$; all barrier shapes appear to fit our data equally well.

It is therefore gratifying that the value obtained for the barrier height proves to be relatively insensitive to the barrier shape: All values fall within a standard deviation from the value obtained for the simple cosine potential, $V_{0}=0.91 \pm 0.32 \mathrm{kcal} \mathrm{mole}^{-1}$. We believe that the reason for this insensitivity is that the barrier is relatively low. It is seen from Figs. 9 and 10 that the prob- 


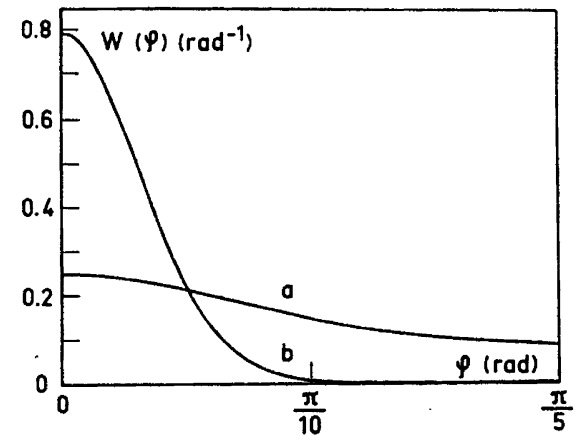

Fig. 8. Angular probability functions of ferrocene. a, $V_{0}=0.809 \mathrm{kcal}$ mole ${ }^{-1}$ and $b$, $V_{0}=7.379 \mathrm{kcal} \mathrm{mole}^{-1}$. $T=413^{\circ} \mathrm{K}$.

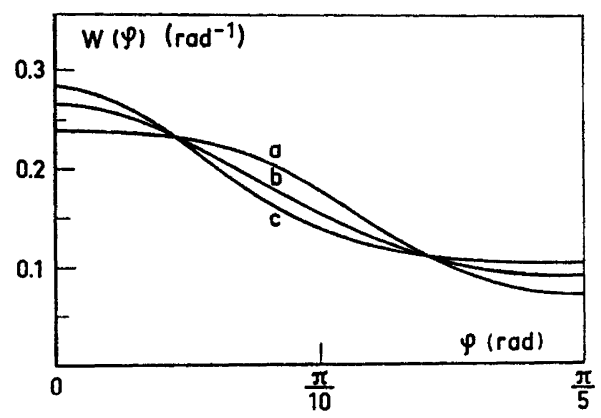

Fig. 9. Angular probability functions ob. tained by least-squares refinement of $W_{1}$ and $u_{1}$.

a, $\beta_{1}=-0.25 ; V_{0}=0.99 \pm 0.39 \mathrm{kcal} \mathrm{mole}^{-1}$; $\mathrm{b}, \quad \beta_{1}=0.00 ; V_{0}=0.91 \pm 0.32 \mathrm{kcal} \mathrm{mole} \mathrm{m}^{-1}$ c, $\beta_{1}=0.25 ; \quad V_{0}=0.81 \pm 0.28 \mathrm{kcal} \mathrm{mole}^{-1}$.

ability of the molecule being antiprismatic is by no means negligible. If the barrier was so high that all molecules were in the immediate vicinity of the prismatic conformation, $\ln W(\varphi)$ would be accurately determined only in the region near $\varphi=0^{\circ}$, while the magnitude at $\varphi=36^{\circ}$, and hence the barrier height, would be based on a highly uncertain and shape-dependent extrapolation.

When computing the barrier heights we have assumed that the temperature of the gas in the scattering region is equal to the temperature of the solid silver nozzle. This is not strictly correct, as the gas expands into the vacuum chamber its temperature drops. ${ }^{38}$ Hence the nozzle temperature is an upper

Table 4. Determination of the barrier to internal rotation in ferrocene. $W(\varphi)=W_{8}(\varphi)$ (see the text). $u_{\mathrm{f}}(\varphi)=C_{1}+D_{1} \cos \varphi$. The result of simultaneous least-squares refinement of $A_{2}, C_{1}$, and $D_{1}$ for a series of values of $\beta_{2}$.

\begin{tabular}{|c|c|c|c|c|c|c|}
\hline$\beta_{2}$ & $\begin{array}{c}\text { Square } \\
\text { error sum }\end{array}$ & $A_{2}$ & $C_{1}(\AA)^{a}$ & $D_{1}(\AA)^{a}$ & $V_{0} / k T$ & $\left.V_{0}(\mathrm{kcal} \mathrm{mole})^{-1}\right)$ \\
\hline 1.5 & 2.09 & 0.55 & 0.110 & -0.003 & $1.27 \pm 0.66$ & $1.04 \pm 0.54$ \\
\hline 2.0 & 2.09 & 0.50 & 0.110 & -0.003 & $1.08 \pm 0.55$ & $0.89 \pm 0.45$ \\
\hline 2.5 & 2.09 & 0.48 & 0.110 & -0.003 & $1.02 \pm 0.51$ & $0.84 \pm 0.42$ \\
\hline 3.0 & 2.09 & 0.46 & 0.110 & -0.003 & $0.99 \pm 0.50$ & $0.81 \pm 0.41$ \\
\hline 4.0 & 2.09 & 0.45 & 0.110 & -0.003 & $0.94 \pm 0.48$ & $0.77 \pm 0.39$ \\
\hline 5.0 & 2.09 & 0.45 & 0.110 & -0.003 & $0.94 \pm 0.48$ & $0.77 \pm 0.39$ \\
\hline 7.0 & 2.09 & 0.44 & 0.110 & -0.003 & $0.93 \pm 0.46$ & $0.76 \pm 0.38$ \\
\hline 9.0 & 2.09 & 0.44 & 0.110 & -0.003 & $0.93 \pm 0.46$ & $0.76 \pm 0.38$ \\
\hline 12.0 & 2.09 & 0.44 & 0.110 & -0.003 & $0.91 \pm 0.46$ & $0.75 \pm 0.38$ \\
\hline 15.0 & 2.09 & 0.44 & 0.110 & -0.003 & $0.91 \pm 0.46$ & $0.75 \pm 0.38$ \\
\hline
\end{tabular}

${ }^{a}$ Standard deviation $=0.007-0.008 \AA$. 
Table 5. Determination of the barrier to internal rotation in ferrocene. $W(\varphi)=N$ $\exp \left(A_{1} \cos 5 \varphi\right)$ and $u_{\mathrm{f}}(\varphi)=R(\varphi)^{-1}\left(C_{2}+D_{2} \cos \varphi+\gamma \cos ^{2} \varphi\right)^{\frac{1}{2}}$. The result of simultaneous least-squares refinement of $A_{1}, C_{2}$, and $D_{2}$ for a series of values of $\gamma$.

\begin{tabular}{rcccccc}
$\gamma\left(\AA^{4}\right)$ & $\begin{array}{c}\text { Square } \\
\text { error sum }\end{array}$ & $A_{1}$ & $C_{2}\left(\AA^{4}\right)^{a}$ & $D_{2}\left(\AA^{4}\right)^{a}$ & $V_{0} / k T$ & $V_{0}\left(\mathrm{kcal} \mathrm{mol \theta ^{-1 }}\right)$ \\
\hline-0.03 & 2.06 & 0.69 & 0.20 & -0.05 & $1.36 \pm 0.52$ & $1.12 \pm 0.43$ \\
-0.02 & 2.07 & 0.67 & 0.19 & -0.05 & $1.32 \pm 0.50$ & $1.08 \pm 0.41$ \\
-0.01 & 2.08 & 0.63 & 0.18 & -0.05 & $1.26 \pm 0.48$ & $1.03 \pm 0.39$ \\
0.00 & 2.09 & 0.61 & 0.17 & -0.05 & $1.21 \pm 0.45$ & $0.99 \pm 0.37$ \\
0.01 & 2.10 & 0.58 & 0.16 & -0.05 & $1.15 \pm 0.43$ & $0.94 \pm 0.35$ \\
\hline
\end{tabular}

$a_{\text {Standard deviation }}=0.02 \AA^{4}$.

limit for the (vibrational) temperature of the gas at the diffraction point, and the value obtained for $V_{0}$ may be too high.

It is seen that the values of the parameters $C_{1}$ and $D_{1}$ also are quite independent of the barrier shape. $u_{\mathrm{f}}(\varphi)$ is plotted in Fig. 11, curve c.

It remains to be seen if the barrier height obtained is sensitive to the form assumed for the framework vibrational amplitude. Recently Cyvin and coworkers ${ }^{39}$ have argued that in ethane-like molecules $u_{\mathrm{f}}(\varphi)$ should be closely approximated by

$$
u_{2}(\varphi)=(1 / R(\varphi))\left(C_{2}+D_{2} \cos \varphi+\gamma \cos ^{2} \varphi\right)^{\frac{2}{2}}
$$

Least-squares refinement was therefore carried out with this expression for $u_{\mathrm{f}}(\varphi)$ along with the simple cosine form of the potential. $A_{1}, C_{2}$, and $D_{2}$ were refined simultaneously for a series of values of $\gamma$. The results are listed in Table 5, three of the functions obtained for the framework vibration are

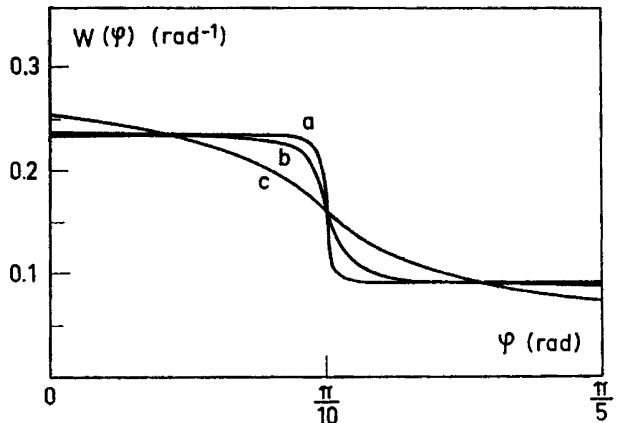

Fig. 10. Angular probability functions obtained by least-squares refinement of $W_{2}$ and $u_{1}$.

a, $\beta_{2}=15.0 ; V_{0}=0.75 \pm 0.38 \mathrm{kcal}$ mole $^{-1}$;

b, $\beta_{2}=4.0 ; V_{0}=0.77 \pm 0.39 \mathrm{kcal} \mathrm{mole}^{-1}$;

c, $\beta_{2}=1.5 ; \quad V_{0}=1.04 \pm 0.54$ kcal mole m. $^{-1}$.

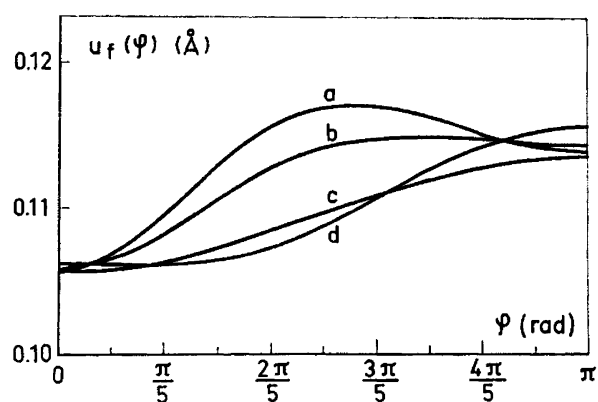

Fig. 11. Framework vibrational amplitudes obtained by refinement on $W_{1}\left(\beta_{2}=0\right)$ and different $u_{\mathrm{f}}(\varphi)$ 's.

a, $u_{2}(\varphi)\left(\gamma=-0.02 \AA^{4}\right), V_{0}=1.08 \pm 0.41 \mathrm{kcal}$ $\mathrm{mole}^{-1} ; \mathrm{b}, u_{2}(\varphi), V_{0}=1.03 \pm 0.39 \mathrm{kcal} \mathrm{mole}^{-1}$; c, $u_{1}(\varphi)\left(\gamma=-0.01 \AA^{4}\right), V_{0}=0.91 \pm 0.32 \mathrm{kcal}$ mole $^{-1}$; d, $u_{2}(\varphi)\left(\gamma=0.01 \AA^{4}\right), V_{0}=0.94 \pm 0.35$ kcal mole ${ }^{-1}$.

Acta Chem. Scand. 22 (1968) No. 8 
plotted in Fig. 11. The curves differ greatly in the intermediate range, but at $\varphi=0$ and $\varphi=\pi$ they fall within a standard deviation of the values obtained by refinement on $u_{1}(\varphi), u_{1}(0)=0.106 \pm 0.011 \AA$, and $u_{1}(\pi)=0.114 \pm 0.011 \AA$. More important, even though the curves are very different, the barrier heights fall within a standard deviation of the one obtained by refinement on $u_{1}(\varphi)$.

In order to assure that no systematic errors have been introduced by the neglect of shrinkage ${ }^{40}$ in our calculations, a final refinement was carried out with the inter-ring distance $H$ as an independent parameter along with $A_{1}, C_{1}$, and $D_{1}$. The resulting value for $H(3.357 \pm 0.007 \AA)$ was actually higher than the value computed from the $\mathrm{C}-\mathrm{C}$ and $\mathrm{Fe}-\mathrm{C}$ bond distances $(3.322 \pm 0.002 \AA)$. The square error sum decreased considerably (to 1.90), and the barrier height obtained was $0.81 \pm 0.26 \mathrm{kcal} \mathrm{mole}^{-1}$.

Least-squares calculations on ruthenocene were much less successful. Refinement on $u_{1}(\varphi)$ and the simple cosine form of the potential gave $V_{0}=26 \pm 49 \mathrm{kcal} \mathrm{mole}^{-1}$. The reason for this failure is undoubtedly that the molecular intensity, due to the heavy background of atomic scattering from $\mathrm{Ru}$, was not as accurately determined as for ferrocene. Also the contribution from the C... $\mathrm{C}^{\prime}$ distances to the molecular intensity is relatively smaller than in ferrocene.

\section{CONCLUDING REMARKS}

It is difficult not to draw the conclusion from the calculations in the last paragraph that the equilibrium conformation of the free ferrocene molecule is prismatic and that the barrier to internal rotation is $V_{0}=0.9 \pm 0.3 \mathrm{kcal} \mathrm{mole}^{-1}$.

This barrier is so low, that in the gas phase at room temperature and above a considerable fraction of ferrocene molecules are in or near the antiprismatic configuration.

Also the barrier height is so much smaller than the heat of sublimation (16.29 kcal mole $\mathrm{k}^{-1}$ ) that the configuration in the crystal easily could be determined by packing forces.

The origin of the barrier can at this point only be a matter of speculation. It could conceivably be due to some aspect of the ligand-to-metal-to-ligand bonding or to van der Waals' attraction between the rings. In the former case, since ruthenocene is more strongly bonded than ferrocene, one might expect a higher barrier in that compound. (The heat of the reaction $\mathrm{M}(\mathrm{g})+2 \mathrm{C}_{5} \mathrm{H}_{5}(\mathrm{~g}) \rightarrow \mathrm{M}\left(\mathrm{C}_{5} \mathrm{H}_{5}\right)_{2}$ (g) has been estimated to $-187 \mathrm{kcal}$ mole ${ }^{-1} \mathbf{4 2}^{2}$ for $\mathrm{M}=\mathrm{Ru}$, to $-147 \mathrm{kcal} \mathrm{mole}^{-143}$ for $\mathrm{M}=\mathrm{Fe}$ ). This in turn might explain why ruthenocene retains the prismatic shape in the crystalline environment.

Acknowledgement. Wo are grateful to the Norwegian Research Council for Science and the Humanities for financial support, and to professor Jack Dunitz, professor Otto Bastiansen, and Dr. H. M. Seip for many helpful discussions. 


\section{REFERENCES}

1. Miller, S. A., Tebboth, J. A. and Tremaine, J. F. J. Chem. Soc. 1952632.

2. Kealy, T. J. and Pauson, P. L. Nature 168 (1951) 1039.

3. Fischer, E. O. and Phab, W. Z. Naturforsch. 76 (1952) 377.

4. Wilkinson, G., Rosenblum, M., Whiting, M. C. and Woodward, R. B. J. Am. Chem. Soc. 74 (1952) 2125.

5. Dunitz, J. D., Orgel, L. E. and Rich, A. Acta Cryst. 9 (1956) 373.

6. Willis, B. T. AERE Report (Harwell) R3708 (1961).

7. Edwards, J. W., Kington, G. L. and Mason, R. Trans. Faraday Soc. 56 (1960) 660.

8. Kaluski, Z. L., Struchkov, Yu. T. and Avoyan, R. L. J. Struct. Chem. 5 (1964) 683.

9. Macdonald, A. C. and Trotter, J. Acta Cryst. 17 (1964) 872.

10. Kaluski, Z. L. and Struchkov, Yu. T. J. Struct. Chem. 6 (1965) 296.

11. Hohn, C. H. and Ibers, J. A. J. Chem. Phys. 30 (1959) 885.

12. Hardgrave, G. L. and Templeton, D. H. Acta Cryst. 12 (1959) 28.

13. Lippincott, E. R. and Nelson, R. D. Spectrochim. Acta 10 (1958) 307.

14. Stammreich, H. quoted by Fritz in Ref. 15.

15. Fritz, H. P. In Advan. Organomet. Chem. 1 (1964).

16. Seibold, E. A. and Sutton, L. E. J. Chem. Phys. 23 (1955) 1967.

17. Akishin, P. A., Rambidi, N. G. and Bredikhina, T. N. J. Struct. Chem. 2 (1961) 443.

18. Bohn, R. K. and Haaland, A. J. Organomet. Chem. 5 (1966) 470.

19. Bastiansen, O., Hassel, O. and Risberg, E. Acta Chem. Scand. 9 (1955) 232.

20. Almenningen, A., Bastiansen, O., Haaland, A. and Seip, H. M. Angew. Chem. 77 (1965) 877.

21. Peacher, J. L. and Wills, J. C. J. Chem. Phys. 46 (1967) 4807.

22. Clementi, E., Roothan, C. C. J. and Yoshimine, M. Phys. Rev. 127 (1962) 1618.

23. Watson, R. E. Phys. Rev. 119 (1960) 1934.

24. Strand. T. G. and Bonham, R. A. J. Chem. Phys. 40 (1964) 1686.

25. Liberman, D., quoted by Cox and Bonham in Ref. 26.

26. Cox, H. L. and Bonham, R. A. J. Chem. Phys. 47 (1967) 2599.

27. Hedberg, K. and Iwasaki, M. Acta Cryst. 17 (1964) 529.

28. Almenningen, A., Bastiansen, O., Seip, R. and Seip, H. M. Acta Chem. Scand. 18 (1964) 2115.

29. Hamilton, W. C. Acta Cryst. 18 (1965) 502.

30a. Haaland, A. Acta Chem. Scand. 19 (1965) 41.

30b. Haaland, A. and Nilsson, J.-E. Unpublished result.

31. Almenningen, A., Haaland, A. and Motzfeldt, T. In Selected Topics in Structure Chemistry, Universitetsforlaget, Oslo 1967.

32. Almenningen, A., Haaland, A. and Motzfeldt, T. J. Organomet. Chem. 7 (1967) 97.

33. Bartell, L. S. J. Chem. Phys. 23 (1955) 1219.

34. Merzbacher, E. Quantum Mechanics, Wiley, New York 1961, p. 77.

35. Morino, Y. and Hirota, E. J. Chem. Phys. 28 (1958) 185.

36. Nielsen, H. H. Phys. Rev. 40 (1932) 445.

37. Goldstein, S. Trans. Com. Phil. Soc. 23 (1927) 303.

38. Hagena, O. and Henkes, W. Z. Naturforsch. 15a (1960) 851.

39. Cyvin, S. J., Elvebredd, I., Cyvin, B. N., Brunvoll, J. and Hagen, G. Acta Chem. Scand. 21 (1967) 2405.

40. Bastiansen, O. and Skancke, P. N. In Advan. Chem. Phys. 3 (1960) 359.

41. Edwards, J. W. and Kington, G. L. Trans. Faraday Soc. 58 (1962) 1323.

42. Fischer, E. O. and Grubert, H. Chem. Ber. 92 (l d d) 2302.

43. Wilkinson, G., Pauson, P. L. and Cotton, F. A J. Am. Chem. Soc. 76 (1954) 1970.

Received March 28, 1968. 